\title{
Guest editorial: web information technologies
}

\author{
Xuemin Lin' 1 Jeffrey Xu Yu ${ }^{2}$
}

Published online: 13 June 2015

(C) Springer Science+Business Media New York 2015

The last decades witnessed the rapid growth of Web technologies, methodologies and applications. This special issue collects eight research papers which are extensions of the best papers of the WISE 2013 conference(14 ${ }^{\text {th }}$ International Conference of Web Information Systems Engineering, Nanjing, China).

The first paper, by Huang et al, "Mining streams of short text for analysis of world-wide event evolutions", addresses the limitations of existing techniques such as LDA in detecting and tracking events through short text analysis. The authors propose a novel algorithm named FTCCT (Finding Topic Clusters using Co-occurring Terms) to automatically generate topics from a short text corpus, and develop an Event Evolution Mining (EEM) algorithm to discover hot events and their evolutions. Evolutions of the hot events are also visualized in the paper.

The second paper, by Li et al., "Social event identification and ranking on flickr", focuses on social event modeling and ranking. A new method is introduced to effectively identify events by considering the spreading effect of event in the spatio-temporal space, and a selfexciting point process model is developed to capture the triggering relationships among events. Furthermore, event impact is defined and estimated via random walk based on the triggering relationships.

Huang et al. study user attribute identification or profile inference in the third paper, "A multi-source integration framework for user occupation inference in social media systems". The authors propose a comprehensive framework to infer the user occupation from his/her social activities recorded in the micro-blog system, by identifying some beneficial content features and proposing a machine learning classification model.

\section{Xuemin Lin}

1xue@cse.unsw.edu.au

Jeffrey Xu Yu

yu@se.cuhk.edu.hk

1 The University of New South Wales, Sydney, Australia

2 Chinese University of Hong Kong, Shatin, Hong Kong 
In the fourth paper, "User communities evolution in microblogs: A public awareness barometer for real world events", Giatsoglou, Chatzakou and Vakali study the problem of user communities evolution in microblogs. The proposed community detection approach identifies communities exploiting both structural properties and intensity patterns, while dynamics of communities evolution around an event are revealed based on an iterative community detection and mapping scheme.

In the fifth paper, "Multi-relational PageRank for Tree Structure Sense Ranking", Interdonato and Tagarelli study the problem of structural sense ranking for tree data using a multi-relational PageRank approach. The authors developed the first PageRank-based formulations that exploit heterogeneity of links to address the problem of structural sense ranking in tree data.

Zhang et al. study skyline processing in probabilistic streams in the sixth paper, "Probabilistic n-of-N Skyline Computation over Uncertain Data Streams". The paper firstly identifies a candidate set with the minimum number of uncertain elements to be kept. Then efficient query processing and index structure updating techniques are developed based on R-tree structures.

Yao et al. address the limitations of recommendation systems in existing techniques and cope with scenarios with only implicit feedback in the seventh paper, "A Graph-based Model for Context-aware Recommendation using Implicit Feedback Data". The authors propose a graph-based generic recommendation framework, which constructs a Multi-Layer Context Graph (MLCG) from implicit feedback data, and performs ranking algorithms in MLCG for context-aware recommendation.

Finally, the paper "Detecting Overlapping Communities in Poly-Relational Networks", by $\mathrm{Wu}$ et al., focuses on discovering overlapping communities. The authors relax strong assumptions held by existing techniques and introduce a novel co-ranking framework named MutuRank. MutuRank makes full use of the mutual influence between relations and actors to transform the poly-relational network to the mono-relational network.

We wish to thank the authors for their contributions in this special issue. We also thank the reviewers for their valuable comments throughout the rigours review phases. We hope these papers provide the community with insights and inspirations for continued research work. 